INPLASY

PROTOCOL

To cite: Wang et al. Superb microvascular imaging in guiding targeted biopsy of prostate Cancer: A protocol for systematic review and meta analysis. Inplasy protocol

2020100117. doi:

10.37766/inplasy2020.10.0117

Received: 31 October 2020

Published: 31 October 2020

Corresponding author:

Cong Wang

wc027214@163.com

Author Affiliation:

The First Affiliated Hospital of Dalian Medical University

Support: LZ2020027 (Liao Ning).

Review Stage at time of this submission: Preliminary searches.

Conflicts of interest:

None.

\section{Superb microvascular imaging in guiding targeted biopsy of prostate Cancer: A protocol for systematic review and meta analysis}

\author{
Wang, C1; Tao, Y2.
}

Review question / Objective: The present meta-analysis aimed at determining the accuracy of SMI in the location diagnosis for prostate cancer.

Condition being studied: Studies suggested SMI technology to guide prostate cancer biopsy could improve the positive rate of draw materials. However, there is no systematic review or meta-analysis providing evidence to determine whether SMI is an ideal method to guide prostate cancer biopsy. Information sources: PubMed, Web of Science, Cochrane Library, and Chinese biomedical databases will be searched from their inceptions to the October 31st, 2020.

INPLASY registration number: This protocol was registered with the International Platform of Registered Systematic Review and Meta-Analysis Protocols (INPLASY) on 31 October 2020 and was last updated on 31 October 2020 (registration number INPLASY2020100117).

\section{INTRODUCTION}

Review question / Objective: The present meta-analysis aimed at determining the accuracy of SMI in the location diagnosis for prostate cancer.
Condition being studied: Studies suggested SMI technology to guide prostate cancer biopsy could improve the positive rate of draw materials. However, there is no systematic review or meta-analysis providing evidence to determine whether SMI is an ideal method to guide prostate cancer biopsy. 


\section{METHODS}

Participant or population: The patients should be those who undergone prostate biopsy.

Intervention: SMI.

Comparator: Pathology.

Study designs to be included: This study will only include high quality clinical cohort or case control studies.

Eligibility criteria: Type of study. This study will only include high quality clinical cohort or case control studies. Type of patients. The patients should be those who undergone prostate biopsy. We will not apply any restrictions of race, age, education background, and economic status. Intervention and comparison. This study compare SMI with pathology for diagnosing prostate cancer. Type of outcomes. The primary outcomes include sensitivity, specificity, positive and negative likelihood ratio, diagnostic odds ratio, and the area under the curve of the summary receiver operating characteristic.

Information sources: PubMed, Web of Science, Cochrane Library, and Chinese biomedical databases will be searched from their inceptions to the October 31st, 2020.

Main outcome(s): The primary outcomes include sensitivity, specificity, positive and negative likelihood ratio, diagnostic odds ratio, and the area under the curve of the summary receiver operating characteristic.

Quality assessment / Risk of bias analysis: Methodological quality was independently assessed by two researchers based on the quality assessment of studies of diagnostic accuracy studies (QUADAS) tool.

Strategy of data synthesis: The STATA version 14.0 (Stata Corp, College Station, TX, USA) and Meta-Disc version 1.4 (Universidad Complutense, Madrid, Spain) softwares were used for meta-analysis. We calculated the pooled summary statistics for sensitivity, specificity, positive and negative likelihood ratio, and diagnostic odds ratio with their $95 \%$ confidence intervals. The summary receiver operating characteristic curve and corresponding area under the curve were obtained. The threshold effect was assessed using Spearman correlation coefficients.

Subgroup analysis: We also performed sub group and meta-regression analyses to investigate potential sources of heterogeneity.

Sensibility analysis: To evaluate the influence of single studies on the overall estimate, a sensitivity analysis was performed.

Country(ies) involved: China.

Keywords: meta-analysis; prostate cancer; superb microvascular imaging.

Contributions of each author:

Author 1 - Cong Wang.

Author 2 - Ye Tao. 